\title{
The exact status and synonyms of three Chinese Afronurus Lestage, 1924 established by Navás in 1936 (Ephemeroptera: Heptageniidae)
}

\author{
XIAOLI YING ${ }^{1,2} \&$ CHANGFA ZHOU ${ }^{1, *}$ \\ ${ }^{1}$ The Key Laboratory of Jiangsu Biodiversity and Biotechnology, College of Life Sciences, Nanjing Normal University, Nanjing 210023 , \\ China \\ ${ }^{2}$ ="yx19106@126.com; @ https://orcid.org/0000-0002-1808-9993 \\ "Corresponding author. !"zhouchangfa@njnu.edu.cn; ○ https://orcid.org/0000-0001-8785-5228
}

\begin{abstract}
Navás named more than 20 mayfly species from China but most of them are not associated with currently known specimens. In 2021, we found three common species of Chinese Afronurus Lestage, 1924 (Heptageniidae), which genitalia and color patterns match three species described and illustrated by Navás in 1936. Therefore, three new synonyms are established: Afronurus pallescens (Navás, 1936) (=Cinygmina obliquistriata You et al., 1981, syn. nov.), Afronurus kiangsuensis (Puthz, 1971) (renamed from Ecdyonurus hyalinus Navás, 1936= Cinygmina rubromaculata You et al., 1981, syn. nov.), Afronurus costatus (Navás, 1936) (=Cinygmina yixingensis Wu \& You, 1986, syn. nov.). As the type specimens for Navas' three species are supposed to be lost, neotypes are designated for them.
\end{abstract}

Key words: taxonomy, old species, association, China, neotype designation

\section{Introduction}

From 1922 to 1936, Navás described 24 mayfly species from China (Zhou et al. 2015). Because of lost or damaged types (Alba-Tercedor \& Peters 1985), brief original descriptions and inaccurate illustrations, most of them are poorly known, especially those in the families Baetidae, Ephemeridae, Heptageniidae and Leptophlebiidae.

In 2021, after a review of the Chinese Afronurus Lestage, 1924 species (Zhang et al. 2021), we noticed a paper of Navás published in 1936 with eleven mayfies (seven in the family Heptageniidae). His drawings of the abdominal color pattern and genitalia of three heptageniid species, Ecdyonurus pallescens Navás, 1936, Ecdyonurus hyalinus Navás, 1936, and Heptagenia costata Navás, 1936, appear to match those of more recently named species. As the type specimens of Navás species deposited in the Heude Museum (now the Shanghai Entomological Museum) can no longer be located (Alba-Tercedor \& Peters 1985; Prof. Hong Gui, personal communication), we collected specimens from nearby their type localities, allowing us to confirm that they are conspecific with three Afronurus species, which are very common in China and have very wide distribution. So all these species names are listed and clarified here, neotypes are redesigned for the three species. The detailed descriptions, photos, diagnoses and real status of them can be found in Zhang et al. (2021).

All neotypes and other specimens mentioned in this article were collected from China and are deposited in the Mayfly collection, College of Life Sciences, Nanjing Normal University (NJNU), China.

\section{Afronurus pallescens (Navás, 1936)}

Ecdyonurus pallescens Navás, 1936: 121, fig. 81 (male, female). Types: male, female, Kuling, Jiangxi province, China. Ecdyonurus pallescens-Gui 1985: 87.

Cinygmina obliquistriata You et al., 1981: 26, pl. 1, figs 1-13 (male, female). Types: male, female, Yixing, Jiangsu Prov. New Synonym.

Cinygmina obliquistriata-Wu et al. 1986: 65, figs 12-22 (nymph). 
Cinygmina obliquistriata - Gui 1985: 86; Zhang \& Cai 1991: 237; You \& Gui 1995: 51, fig. 50 (male); Su et al. 1995: 41; Gui et al. 1999: 330, figs 11-10 (male); Zhou \& Zheng 2003: 756, figs 9, 12, 16 (male and nymph).

Afronurus obliquistriatus-Braasch \& Jacobus 2011: 65; Zhang et al. 2021: 109.

Remarks: Both imagoes (Figs. 1B, 1C) and nymphs of C. obliquistriata have remarkable oblique abdominal stripes that clearly match the original descriptions and drawings (Fig. 1A) of E. pallescens by Navás (1936). Therefore, we propose the following synonymy: Ecdyonurus pallescens Navás, 1936 (= Cinygmina obliquistriata You et al., 1981). In addition, since the types of this species are lost (Alba-Tercedor \& Peters 1985), we redesign a neotype for it.

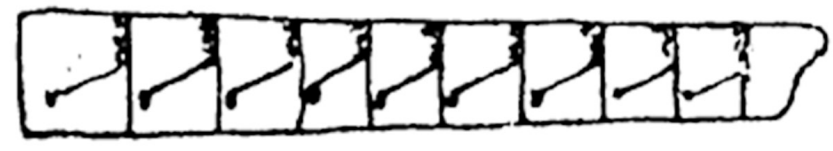

Fig. 81.

Ecdyonurus pallescens $\sigma^{*}$ Nav. A Abdomen, vu de côté.
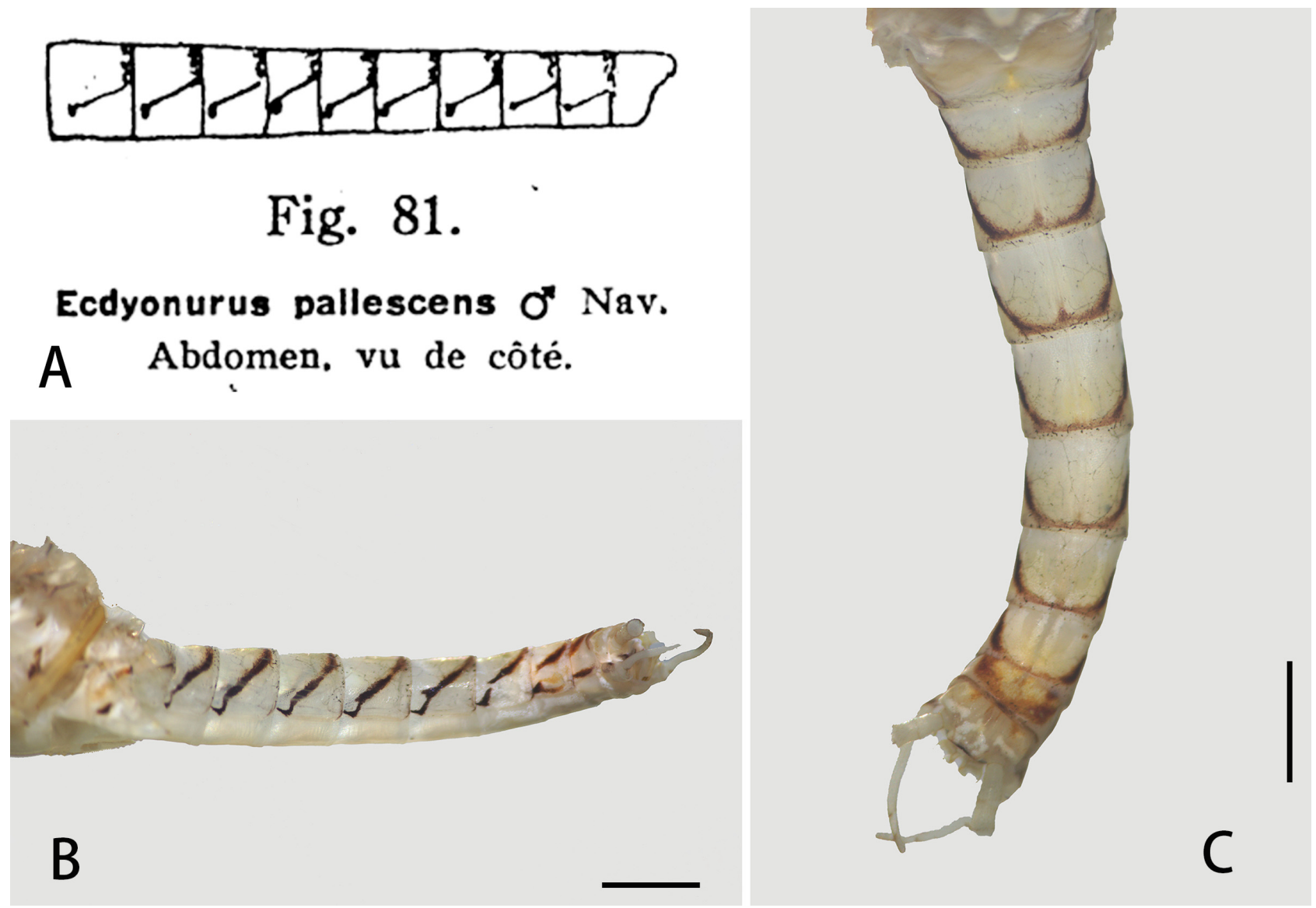

FIGURE 1. Abdomen of Afronurus pallescens (Navás, 1936) male imago. A: Original drawing from Navás (1936). B: Lateral view. C: Dorsal view. Scale bars $=1.0 \mathrm{~mm}$.

Neotype designation for Afronurus pallescens (Navás, 1936): male imago, China, Jiangxi Province, Wu-

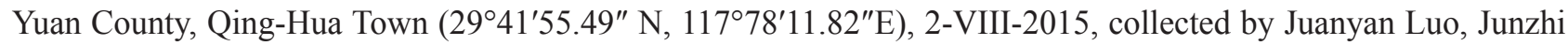
Sun, Yike Han. The neotype designed here was collected from the same province and in a very nearby locality of the original types, and has typical characters mentioned in the original description.

Other material examined: 19 male imagoes, 2 female imagoes, same data as the neotype; male imago (holotype of Cinygmina obliquistriata You et al., 1981), Jiangsu Province, Yi-Xing County, Ming-Ling Town ( $\left.31^{\circ} 10^{\prime} 50.20^{\prime \prime} \mathrm{N}, 119^{\circ} 40^{\prime} 17.52^{\prime \prime} \mathrm{E}\right), 21-31-\mathrm{VII}-1980$, collected by Tian Wu; 12 male imagoes (paratypes), same data as the holotype; 15 male imagoes, 18 female imagoes, 20 nymphs, Zhejiang Province, Lin-An city, Yu-Qian Town, Tianmu Mountain (30³2'11.91" N, 11944'73.71" E), 3-IV-2019, collected by Wei Zhang, Zhenxing Ma; 2 male imagoes, 2 female subimagoes, Hubei Province, Yi-Chang City, Yang-Lin-Qiao Town $\left(30^{\circ} 74^{\prime} 79.01^{\prime \prime} \mathrm{N}\right.$, $110^{\circ} 76^{\prime} 60.72^{\prime \prime}$ E), 13-VIII-2019, collected by Ran Li, Wei Zhang; 20 male imagoes, 10 male subimagoes, 14 female imagoes, 10 female subimagoes, 12 nymphs, Hunan Province, Yi-Yang City, An-Hua County, Liu-Bu-Xi Nature Reserve (28 $31^{\prime} 16.95^{\prime \prime}$ N, $110^{\circ} 82^{\prime} 65.41^{\prime \prime}$ E), 17-VIII-2019, collected by Ran Li, Wei Zhang.

Distribution: almost all Chinese mainland except the Qinghai-Tibet Plateau. 
Ecdyonurus hyalinus Navás, 1936: 123, fig. 83 (male, female). Types: male, female, Jiangsu (nec Ecdyurus [sic] hyalinus Ulmer, 1912: 369, 372; nec Esben-Petersen 1916: 6). Renamed as Ecdyonurus kiangsuensis by Puthz 1971: 44.

Cinygmina rubromaculata You et al., 1981: 28, pl. 2, figs 14-24 (male, female). Types: male, female, Yixing, Jiangsu province, China. New Synonym.

Cinygmina rubromaculata-Wu et al. 1986: 65, figs 1-11 (nymph).

Cinygmina rubromaculata - Gui 1985: 86; Zhang \& Cai 1991: 237; You \& Gui 1995: 52, fig. 51 (male); Zhou \& Zheng 2003: 758, figs 7, 13, 17 (male and nymph).

Ecdyonurus rubromaculatus-Tshernova et al. 1986: 114.

Cinygmina hainanensis She et al., 1995: 72, fig. 1 (male, female, male subimago). Types: male, female, subimago, Hainan (Synonymized by Zhou \& Zheng 2003: 758).

Afronurus rubromaculatus-Braasch \& Jacobus 2011: 65; Zhang et al. 2021: 110.

Remarks: The abdominal color pattern of this species is very unique (Fig. 2). Comparison of the drawings of Navás (Fig. 2A) with specimens of C. rubromaculata (Figs. 2B, 2C) shows they are equivalent. The types of them were collected from the same county, Jiangsu province, China. Therefore, we propose the following synonymy: Ecdyonurus kiangsuensis Puthz, 1971 (= Cinygmina rubromaculata You et al., 1981).

A

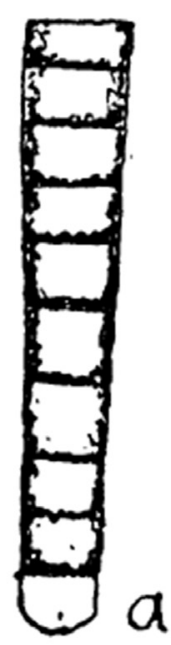

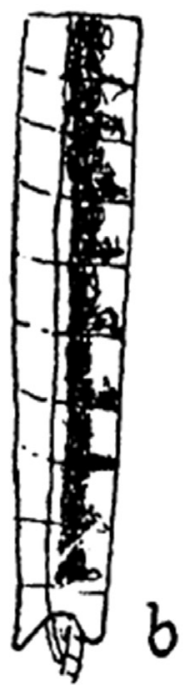

Fig. 83.

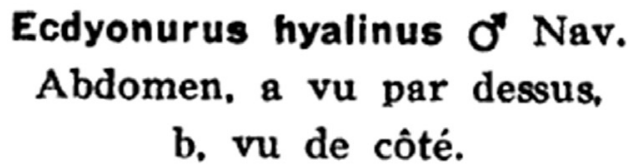

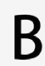

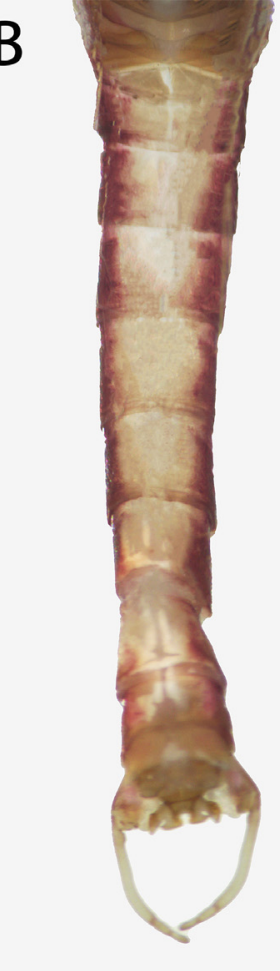

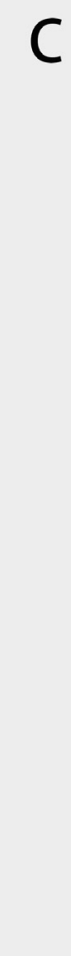

C

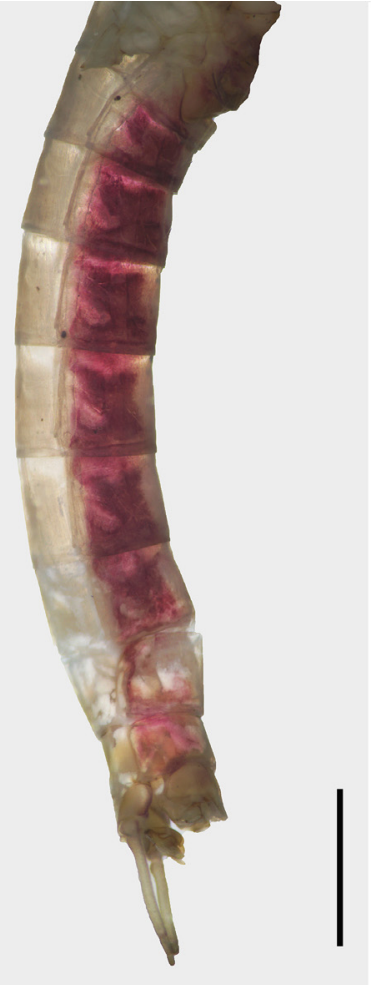

FIGURE 2. Abdomen of Afronurus kiangsuensis (Puthz, 1971) male imago. A: Original drawing from Navás (1936). B: Lateral view. C: Dorsal view. Scale bars $=1.0 \mathrm{~mm}$.

Ulmer (1912) named a species Ecdyurus hyalinus from the Chinese Taiwan island which was later transferred to the genus Afronurus by Kang \& Yang (1994). Ecdyonurus hyalinus Navás, 1936 is therefore a junior homonym and was renamed by Puthz (1971) as Ecdyonurus kiangsuensis. We also redesigned a neotype for this species.

Neotype designation for Afronurus kiangsuensis (Puthz, 1971): male imago, China, Jiangsu Province, Yi-Xing County, Hu-Fu Town (3122'89.41" N, 11979'67.42" E), 18-V-1995, collected by Chaodong Zhu. The neotype designed here was collected from the same province and in a very nearby locality of the original types, and has typical characters mentioned in the original description.

Other material examined: 6 male imagoes, 6 nymphs, same as the neotype; male imago (holotype of Cinygmina rubromaculata You et al., 1981), Jiangsu Province, Yi-Xing County, Ming-Lin Town (3110'5.02" N, $119^{\circ} 40^{\prime} 17.52^{\prime \prime}$ E), 21-VII-1980, collected by Tian Wu; 12 male imagoes, 10 female imagoes (paratypes), same data as holotype; 6 male imagoes, 7 male subimagoes, 10 female imagoes, 8 female subimagoes, 40 nymphs, Hunan 


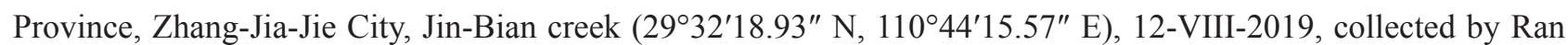
Li, Wei Zhang; 20 male imagoes, 5 male subimagoes, 8 female imagoes, 6 female subimagoes, Hubei Province, YiChang City, Chang-Yang County, Gao-Jia-Yan Town, Qing-Yan Village (3059'41.08" N, 111'11'37.93" E), 20-VII2013, collected by Yanxia Wang, Dan Zhou; 100 nymphs, Hainan Province, Chang-Jiang County, Ba-Wang-Ling (1906'3761" N, 109¹7'0581" E), 15-I-2015, collected by Junzhi Sun, Yike Han.

Distribution: Most areas of Chinese mainland except the Qinghai-Tibet Plateau.

\section{Afronurus costatus (Navás, 1936)}

Heptagenia costata Navás, 1936: 120, fig. 80 (male, female). Types: male, Kuling, Jiangxi province, China. Heptagenia costata-Gui 1985: 84; You \& Gui 1995: 43, fig. 38 (male).

Cinygmina yixingensis Wu \& You, 1986: 280, figs 1-13 (male, female). Types: male, female, Yixing, Jiangsu Prov. New Synonym.

Cinygmina yixingensis - Wu et al. 1986: 66, figs 23-33 (nymph).

Cinygmina yixingensis - Gui 1985: 86; Zhang \& Cai 1991: 237; You \& Gui 1995: 53, fig. 52 (male); She et al. 1995: 79; Zhou $\&$ Zheng 2003: 758, figs 10,14, 18 (adults and nymph).

Afronurus yixingensis-Braasch \& Jacobus 2011: 65; Zhang et al. 2021:111.
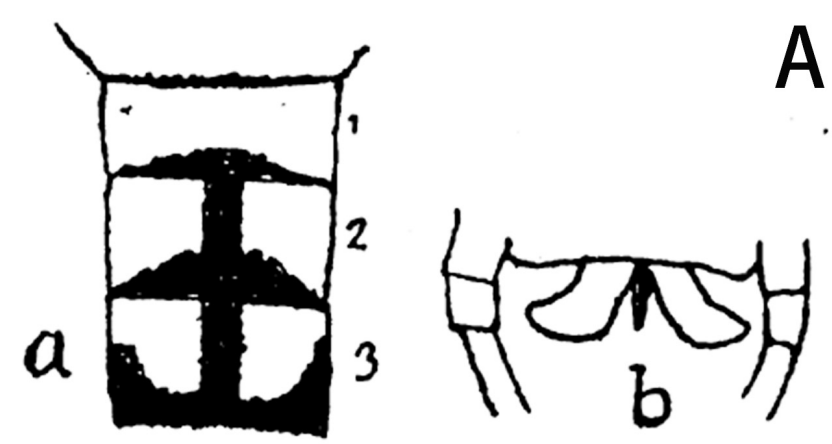

Fig. 80.

- Heptagenia costata on Nav. Abdomen : a Partie antérieure. b Partie postérieure.

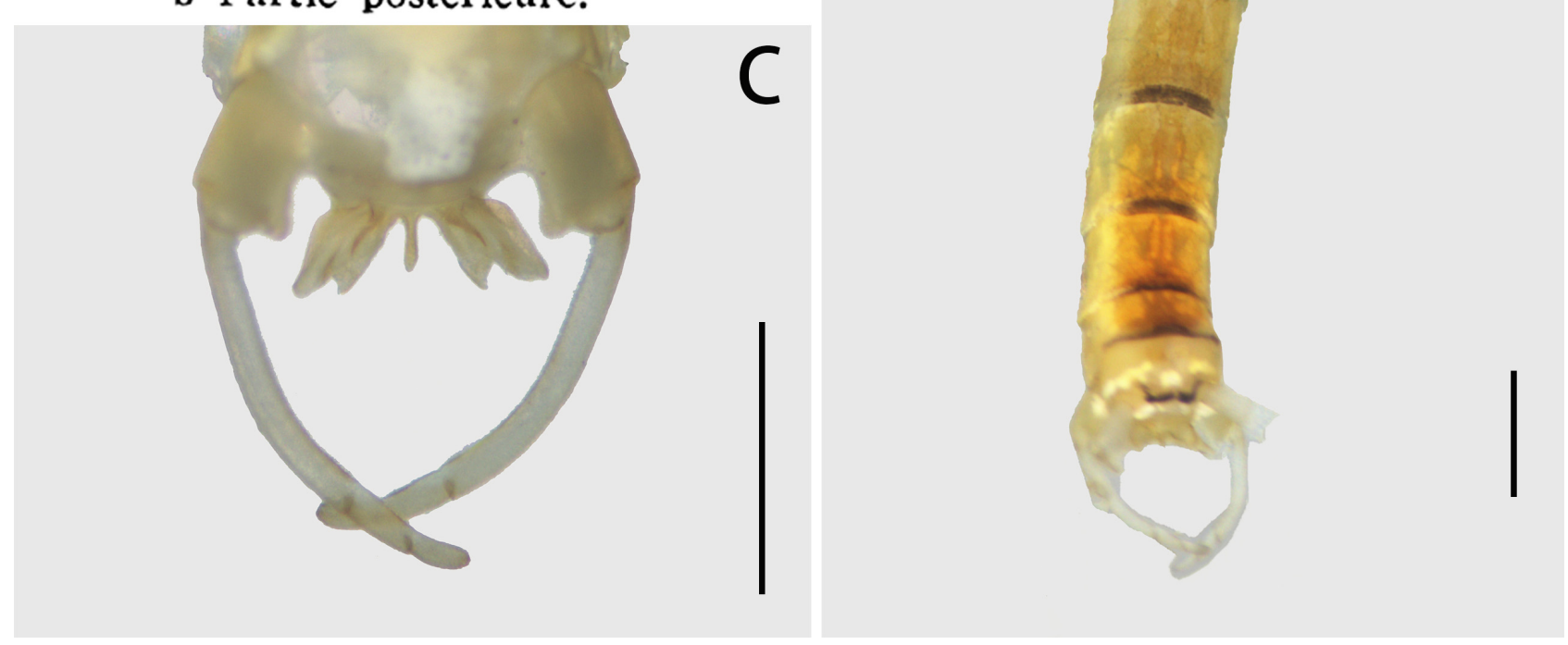

FIGURE 3. Abdomen of Afronurus costatus (Navás, 1936) male imago. A: Original drawing from Navás (1936). B: Dorsal view. C: Genitalia (ventral view). Scale bars: $B=1.0 \mathrm{~mm} ; \mathrm{C}=0.5 \mathrm{~mm}$. 
Remarks: The males of this species have a remarkable projection between the penes (Fig. 3C), which is clearly showed in the original picture of Navás (1936) (Fig. 3A). The color patterns between the original drawing and our fresh materials are also consistent (Figs. 3A, 3B), so we state that the two species established by Navás in 1936 and Wu \& You in 1986 are conspecific. Therefore, we propose the following synonymy: Heptagenia costata Navás, 1936 (= Cinygmina yixingensis $\mathrm{Wu} \&$ You, 1986).

Neotype designation for Afronurus costatus (Navás, 1936): male imago, China, Jiangxi Province, Wu-Yuan

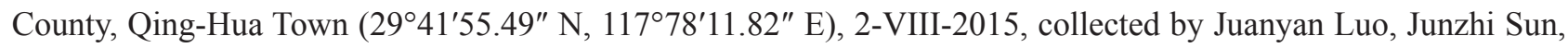
Yike Han. The neotype designed here was collected from the same province and in a very nearby locality of the original types, and has typical characters mentioned in the original description.

Other material examined: 8 male imagoes, 2 female imagoes, same as the neotype; male imago (holotype of Cinygmina yixingensis Wu \& You, 1986), Jiangsu Province, Yi-Xing City, Ming-Lin Town $\left(31^{\circ} 10^{\prime} 5.02^{\prime \prime} \mathrm{N}\right.$, $119^{\circ} 40^{\prime} 17.52^{\prime \prime}$ E), 25-VII-1980, collected by Tian Wu; 10 male imagoes, 2 male subimagoes, 6 female imagoes (paratypes), same as the holotype; 12 male imagoes (some reared from mature nymphs), 15 female subimagoes, 40 nymphs, Hainan Province, Ba-Wang-Ling National Forest Park (1906'37.61" N, 109 $17^{\prime} 05.81^{\prime \prime}$ E), 12-IX2014, collected by Qin Si, Junzhi Sun, Juanyan Luo; 100 nymphs, Anhui Province, Huang-Shan City, Tang-Kou

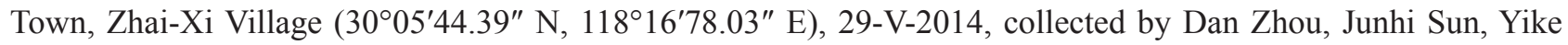
Han; 6 male imagoes, 7 female imagoes, Zhejiang Province, Long-Quan City, Long-Nan Town, Shuang-Xi Village

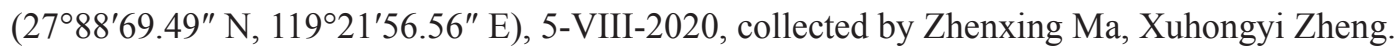

Distribution: Most areas of Chinese mainland except the Qinghai-Tibet Plateau.

\section{References}

Alba-Tercedor, J. \& Peters, W.L. (1985) Types and additional specimens of Ephemeroptera studied by Longinos Navás in the Museo de Zoologia del Ayuntamiento, Barcelona, Spain. Aquatic Insects, 7 (4), 215-227. https://doi.org/10.1080/01650428509361222

Braasch, D. \& Jacobus, L.M. (2011) Two new species of Afronurus Lestage, 1924, from Hong Kong, China (Ephemeroptera: Heptageniidae). Zootaxa, 3062 (1), 64-68.

https://doi.org/10.11646/zootaxa3062.1.7

Esben-Petersen, P. (1916) Ephemerida. In: Resultas scientifiques de'expedition des freres Kusnecov a l'Oural Artique en 1909, sous la direction de H. Backlund. Memoirs of the Imperial Academy of Sciences, Series 8, Section of Physics and Mathematics, 28 (12), 1-12.

Gui, H. (1985) A catalog of the Ephemeroptera of China. Journal of Nanjing Normal University (Natural Science), 4, $79-97$.

Gui, H., Zhou, C.F. \& Su, C.R. (1999) Ephemeroptera. In: Huang, B.K. (Ed.), Insect fauna of Fujian Province. Vol. 1. Fujian Science and Technique Press, Fuzhou, pp. 324-346.

Kang, S.C. \& Yang, C.T. (1994) Heptageniidae of Taiwan (Ephemeroptera). Journal of Taiwan Museum, 47 (1), 5-36.

Navás, L. (1936) Névroptères et insectes voisins. Chine et pays environnants. $9^{\mathrm{e}}$ Série, suite. Notes d'Entomologie Chinoise, Musée Heude, 3 (7), 117-132.

Puthz, V. (1971) Namensänderung einer Heptageniidenart (Ephemeroptera). Mitteilungen der Deutschen Entomologischen Gesellschaft, 30 (4), 44-45. https://doi.org/10.1002/mmnd.4820300405

She, S.S., Gui, H. \& You, D.S. (1995) A research on the mayflies from Hainan province, China (Insecta: Ephemeroptera). Journal of Nanjing Normal University (Natural Science), 18 (2), 72-82.

Su, C.R., Zhou, C.F. \& Gui, H. (1995) Ephemeroptera. In: Wu, H. (Ed.), Insects of Baishanzu Mountain. Eastern China. China Forestry Publishing House, Beijing, pp. 39-43. [in Chinese]

Tshernova, O.A. \& Kluge, N.J., Sinitshenkova N.D. \& Belov V.V. (1986) Identification of Insects of Far East USSR. Vol. 1. Order Ephemeroptera. Leningrad Press, Leningrad [St. Petersburg], pp. 99-142.

Ulmer, G. (1912) H. Sauter's Formosa-Aubsbeute. Ephemeriden. Entomologische Mitteilungen, 1, 369-375. https://doi.org/10.5962/bhl.part.25902

Wu, T. \& You, D.S. (1986) A new species of the genus Cinygmina from China (Ephemeroptera: Ecdyonurinae). Acta Zootaxonomica Sinica, 11, 280-282.

Wu, T., Chen, C.F., Cong, N. \& You, D.S. (1986) Three species of nymphs of the genus Cinygmina from Yi-Xing. Journal of Nanjing Normal University (Natural Science), 1, 65-70.

You, D.S. \& Gui, H. (1995) Economic Insect Fauna of China. Vol. 48: Ephemeroptera. Science Press, Beijing, 152 pp.

You, D.S., Wu, T., Gui, H. \& Hsu, Y.C. (1981) Two new species and diagnostic characters of the genus Cinygmina (Ephemeroptera: Ecdyonurinae). Journal of Nanjing Normal College (Natural Science), 3, $26-32$.

Zhang, J. \& Cai, W.D. (1991) Notes on the genus Cinygmina (Ephemeroptera: Heptageniidae) from Hunan Province, China. Entomotaxonomia, 13, 237-239. 
Zhang, W., Lei, Z.M., Li, W.J. \& Zhou, C.F. (2021)A contribution to the genus Afronurus Lestage, 1924 in China (Ephemeroptera: Heptageniidae, Ecdyonurinae). European Journal of Taxonomy, 767 (1), 94-116.

https://doi.org/10.5852/ejt.2021.767.1491

Zhou, C.F. \& Zheng, L.Y. (2003) The genus Cinygmina (Ephemeroptera: Heptageniidae) in China, with a description of a new species. Acta Entomologica Sinica, 46, 755-760.

https://doi.org/10.16380/j.kcxb.2003.06.015

Zhou, C.F., Su, C.R. \& Gui, H. (2015) Outline of Chinese mayflies. Science Press, Beijing, 310 pp. [in Chinese] 